\title{
Functional Studies of Five Toxin-Antitoxin Modules in Mycobacterium tuberculosis H37Rv
}

\author{
Yoonji Kimt, Eunsil Choit and Jihwan Hwang * \\ Department of Microbiology, Pusan National University, Busan, Republic of Korea
}

Toxin-antitoxin (TA) systems, which consist of an intracellular toxin and its antidote (antitoxin), are encoded by ubiquitous genetic modules in prokaryotes. Commonly, the activity of a toxin is inhibited by its antitoxin under normal growth conditions. However, antitoxins are degraded in response to environmental stress, and toxins liberated from antitoxins consequently induce cell death or growth arrest. In free-living prokaryotes, TA systems are often present in large numbers and are considered to be associated with the adaptation of pathogenic bacteria or extremophiles to various

OPEN ACCESS

Edited by:

Thomas E. Hanson,

University of Delaware, USA

Reviewed by:

Harold J. Schreier,

University of Maryland, Baltimore

County (UMBC), USA

Paras Jain,

Albert Einstein College of Medicine,

USA

*Correspondence:

Jihwan Hwang

hwangjh@pusan.ac.kr

tThese authors have contributed equally to this work.

Specialty section:

This article was submitted to Microbial Physiology and Metabolism,

a section of the journal

Frontiers in Microbiology

Received: 11 August 2016 Accepted: 07 December 2016 Published: 21 December 2016

Citation:

Kim Y, Choi E and Hwang J (2016)

Functional Studies of Five

Toxin-Antitoxin Modules

in Mycobacterium tuberculosis

H37Rv. Front. Microbiol. 7:2071.

doi: 10.3389/fmicb.2016.02071 unfavorable environments by shifting cells to a slow growth rate. Genomic analysis of the human pathogen Mycobacterium tuberculosis H37Rv (Mtb) revealed the presence of a large number of TA systems. Accordingly, we investigated five uncharacterized TA systems (Rv2019-Rv2018, Rv3697c-Rv3697A, Rv3180c-Rv3181c, Rv0299-Rv0298, and Rv3749c-Rv3750c) of Mtb. Among these, the expression of the Rv2019 toxin inhibited the growth of Escherichia coli, and M. smegmatis and this growth defect was recovered by the expression of the Rv2018 antitoxin. Interestingly, Rv3180c was toxic only in M. smegmatis, whose toxicity was neutralized by Rv3181c antitoxin. In vivo and in vitro assays revealed the ribosomal RNA (rRNA) cleavage activity of the Rv2019 toxin. Moreover, mRNAs appeared to be substrates of Rv2019. Therefore, we concluded that the ribonuclease activity of the Rv2019 toxin triggers the growth defect in E. coli and that the Rv2018 antitoxin inhibits the ribonuclease activity of the Rv2019 toxin.

Keywords: toxin, antitoxin, VapBC, MazEF, Mycobacterium tuberculosis

\section{INTRODUCTION}

Toxin-antitoxin (TA) systems are encoded by ubiquitous genetic modules as an operon in prokaryotes and consist of an intracellular toxin and its antidote (antitoxin) (Ramage et al., 2009). Previously, these systems were known as plasmid addiction modules of large plasmids with low copy numbers (Van Melderen et al., 1994). The control of cell death ( $c c d)$ system was first identified as a TA system in the Escherichia coli F plasmid (Jaffe et al., 1985); it is organized as an operon that contains two genes encoding a toxic protein ( $\mathrm{CcdB}$ toxin) and an antidote protein (CcdA antitoxin). When cells are carrying the F plasmid after division, the $c c d B$ toxin and $c c d A$ antitoxin are simultaneously expressed and form a stable TA (CcdB-CcdA) complex that neutralizes toxin activity. However, when the F plasmid is lost by chance during cell division, the CcdA antitoxins remaining in plasmid-free daughter cells are rapidly degraded by the Lon protease. Consequently, $\mathrm{CcdB}$ toxins released from the $\mathrm{CcdB}-\mathrm{CcdA}$ complex interact with their target, DNA gyrase, 
leading to double-strand DNA breaks and ultimately to cell death. Similarly, other plasmid-borne TA systems have been identified as post-segregational killing systems (Gerdes et al., 1990; Jiang et al., 2002) that play a particularly important role in the maintenance of virulence plasmids in pathogenic bacteria (Hayes, 2003; De la Cruz et al., 2013). However, numerous TA systems have recently been discovered; these are encoded by prokaryotic genomes and are commonly considered stress-responsive genetic modules (Sevin and Barloy-Hubler, 2007; Shao et al., 2011).

At present, TA systems are classified into five types according to the nature and mode of action of the antitoxin that counteracts the activity of the toxin (Unterholzner et al., 2013). Toxins are proteins, and antitoxins are either proteins (Types II, IV, and V) or small RNAs (Types I and III) (Schuster and Bertram, 2013). In the Type I system, RNA antitoxins that are complementary to toxin transcripts bind to toxin mRNAs and arrest the translation of toxin mRNAs (Gerdes et al., 1990; Vogel et al., 2004; Weaver et al., 2004; Kawano et al., 2007; Fozo et al., 2008). The toxins and antitoxins of Type II systems are proteins, and most toxins function as endoribonucleases that target rRNA, mRNA, or tRNA. The antitoxin directly associates with its cognate toxin to antagonize the activity of the latter (Jiang et al., 2002; Zhang Y. et al., 2003; Munoz-Gomez et al., 2005; Liu et al., 2008; Jorgensen et al., 2009; Schumacher et al., 2009; Hallez et al., 2010; Winther and Gerdes, 2011; Zhang and Inouye, 2011; Brzozowska and Zielenkiewicz, 2013). In the Type III system, the toxin protein is inactivated by binding to RNA antitoxin fragments cleaved by the ribonuclease of the toxin (Fineran et al., 2009; Blower et al., 2012). Unlike the Type II system, toxins and antitoxins of the Type IV system do not interact directly, and antitoxins protect the target of the toxin, reversing the effect of the toxin (Masuda et al., 2012a,b). In the Type V system, the ribonuclease activity of the antitoxin can degrade the toxin mRNA (Wang et al., 2012).

Surprisingly, a relatively enormous number (79) of TA systems have been identified in the human pathogen Mycobacterium tuberculosis $\mathrm{H} 37 \mathrm{Rv}(\mathrm{Mtb})$, and notably, unlike the related nonpathogenic mycobacterial species, members of the $M$. tuberculosis complex, M. microti, M. bovis, M. africanum, and $M$. canetti, encode an equally large number of TA systems $(66,65,65$, and 60, respectively) (Ramage et al., 2009). Considering this conservation, the TA systems of pathogenic Mycobacteria may be related to their pathogenesis, persistence, virulence, and biofilm formation. Therefore, to investigate the important role of the TA system of $M t b$, we selected 4 uncharacterized TA systems of the VapBC TA family (Rv2019-Rv2018, Rv3697cRv3697A, Rv3180c-Rv3181c, and Rv3749c-Rv3750c) as well as 1 TA system homologous to the MazEF TA family (Rv0299Rv0298). Among these, we showed that expression of the Rv2019 toxin inhibited the growth of $E$. coli and that this growth defect was recovered by expression of the Rv2018 antitoxin. In vitro studies showed that the ribosomal RNA (rRNA) cleavage activity of the Rv2019 toxin requires a divalent metal ion. Therefore, we concluded that the ribonuclease activity of the Rv2019 toxin triggers a growth defect in E. coli and that the Rv2018 antitoxin inhibits the ribonuclease activity of the Rv2019 toxin.

\section{MATERIALS AND METHODS}

\section{Bacterial Strains and Growth Conditions}

The bacterial strains used in this study are listed in Table $\mathbf{1}$. For all studies, bacterial strains were grown with aeration at $37^{\circ} \mathrm{C}$. E. coli BL21(DE3) was grown in LB or M9 minimal media supplemented with casamino acids and $0.2 \%$ glucose or $0.2 \%$ glycerol. M. smegmatis mc $^{2} 155$ was grown in Middlebrook 7H9 (a liquid medium, Difco) containing $0.2 \%$ glycerol and $0.02 \%$ Tween 80 or Middlebrook 7H10 (a solid agar medium, Difco) containing $0.2 \%$ glycerol. Chloramphenicol $(\mathrm{Cm})$ or ampicillin (Amp) was added to a concentration of $50 \mu \mathrm{g} / \mathrm{ml}$ to cultures of E. coli, and kanamycin was added to a concentration of $50 \mu \mathrm{g} / \mathrm{ml}$ to cultures of E. coli and $25 \mu \mathrm{g} / \mathrm{ml}$ to cultures of M. smegmatis.

\section{Plasmid Construction}

To construct plasmids, toxin and antitoxin genes were amplified from $M t b$ genomic DNA by polymerase chain reaction (PCR). The amplified PCR fragments were subcloned into the SmaI site of pUC19, and the sequences of the amplicons were verified using DNA sequencing. Using DNAs digested by the appropriate restriction enzymes, toxin genes were subsequently cloned into the NdeI-HindIII sites (for Rv2019, Rv3180c, $R v 3697 c$, or $R v 0299$ ) or $N d e \mathrm{I}-\mathrm{XbaI}$ sites (for $R v 3749 c$ ) of the pBAD33 vector containing pA15 origin of replication $(\sim 20$ copies per cell) the arabinose-inducible (araBAD) promoter (Guzman et al., 1995) to generate pBAD33-Rv2019, pBAD33Rv3180c, pBAD33-Rv3697c, pBAD33-Rv0299, and pBAD33Rv3749c. Antitoxin genes were cloned into the NdeI-EcoRI sites of the pET21c vector containing an isopropyl $\beta$-D-1thiogalactopyranoside (IPTG)-inducible T7 promoter to generate pET21c-Rv2018, pET21c-Rv3181c, pET21c-Rv3697A, pET21cRv0298, and pET21c-Rv3750c.

To construct a plasmid expressing a chimeric glutathione S-transferase (GST) Rv2019 protein (pGST-Rv2019), pET21cRv2019 was digested with NdeI and XhoI, and the Rv2019 fragment was ligated into the NdeI-XhoI sites of the pGST vector containing an IPTG-inducible T7-lac promoter. To express the toxins in $M$. smegmatis, pUC19-toxin subclones were digested with $N d e I$ and $X b a I$, and the resulting fragment was ligated into the NdeI-XbaI sites of the pMV306AC vector containing an acetamide-inducible promoter (Stover et al., 1991). To construct Rv2019 mutant clones, the primers listed in Supplementary Table S1 were used to amplify gene fragments, and the resulting DNA fragments harboring mutation were inserted into pBAD33, yielding pBAD33-Rv2019 $24 K$, pBAD33Rv2019 ${ }_{E 41 K}$, and pBAD33-Rv2019 ${ }_{D 98 K}$. All plasmids used in this study are listed in Table $\mathbf{1 .}$

\section{Rv2019-Rv2018 Neutralization Assay}

Overnight cultures of BL21(DE3) harboring compatible plasmid pairs (pBAD33 and pET21c, pBAD33-Rv2019 and pET21c, pBAD33 and pET21c-Rv2018, or pBAD33-Rv2019 and pET21cRv2018) were diluted $10^{2}$-fold in fresh M9 minimal media containing $\mathrm{Cm}$ and Amp, and the diluted cultures were grown to the exponential phase at $37^{\circ} \mathrm{C}$. The cells were harvested by 
TABLE 1 | Bacterial strains and plasmids used in this study.

\begin{tabular}{|c|c|c|}
\hline Strains/Plasmids & Genotypes & Reference \\
\hline BW25113 & E. coli, F-, $\Delta(\operatorname{araD}$-araB)567, $\Delta$ lacZ4787(::rrnB-3), $\lambda-$, rph-1, $\Delta(r h a D-r h a B) 568, ~ h s d R 514$ & E. coli Genetic Resources at Yale CGSC \\
\hline BL21(DE3) & $\begin{array}{l}\text { E. coli, F-, lon-11, } \Delta(\text { ompT-nfrA)885, } \Delta \text { (galM-ybhJ)884, } \lambda \text { DE3 [lacl, lacUV5-T7 gene 1, ind1, } \\
\text { sam7, nin5], } \Delta 46,\left[\mathrm{mal}^{+}\right]_{K-12}\left(\lambda^{S}\right), h s d S 10\end{array}$ & E. coli Genetic Resources at Yale CGSC \\
\hline M. smegmatis $\mathrm{mc}^{2} 155$ & M. smegmatis, ept-1 & Snapper et al., 1988 \\
\hline pBAD33 & araBAD promoter, $\mathrm{pACYC184}$ ori, $\mathrm{Cm}^{\mathrm{R}}$ & Guzman et al., 1995 \\
\hline pET21c & T7 promoter, pBR322 ori, Amp ${ }^{R}$ & Novagen \\
\hline pGST & T7-lac promoter, pBR322 ori, Kan ${ }^{\mathrm{R}}$ & Ogawa et al., 1996 \\
\hline pMV306AC & Acetamide promoter, oriE, $\mathrm{Kan}^{\mathrm{R}}$, E. coli-Mycobacterium shuttle vector & Stover et al., 1991 \\
\hline pBAD33-Rv2019 & Rv2019+ (toxin), pBAD33 & This study \\
\hline pBAD33-Rv2019 & Rv2019 D4K (toxin), pBAD33 & This study \\
\hline pBAD33-Rv2019 ${ }_{E 41 K}$ & $R v 2019_{E 41 K}$ (toxin), pBAD33 & This study \\
\hline pBAD33-Rv2019D98K & Rv2019D98K (toxin), pBAD33 & This study \\
\hline pBAD33-Rv3697c & $R v 3697 c^{+}$(toxin), pBAD33 & This study \\
\hline pBAD33-Rv3180c & Rv3180c $^{+}$(toxin), pBAD33 & This study \\
\hline pBAD33-Rv0299 & Rv0299+ (toxin), pBAD33 & This study \\
\hline pBAD33-Rv3749c & $R v 3749 c^{+}$(toxin), pBAD33 & This study \\
\hline pBAD33-Rv2018 & Rv2018+ (antitoxin), pBAD33 & This study \\
\hline pET21c-Rv2019 & Rv2019+ (toxin), pET21c & This study \\
\hline pET21c-Rv2019His & Rv2019+ (toxin), pET21c, C-terminal His-tag & This study \\
\hline pET21c-Rv2018 & Rv2018+ (antitoxin), pET21c & This study \\
\hline pET21c-Rv3697A & $R v 3697 A^{+}$(antitoxin), pET21c & This study \\
\hline pET21c-Rv3181c & 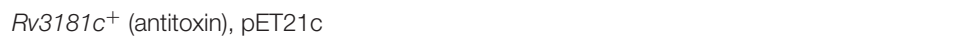 & This study \\
\hline pET21c-Rv0298 & $\mathrm{RV} 298^{+}$(antitoxin), pET21c & This study \\
\hline pET21c-Rv3750c & 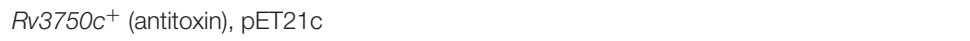 & This study \\
\hline pGST-Rv2019 & Rv2019+ (toxin), pGST & This study \\
\hline pGST-Rv3697c & Rv3697c (toxin), pGST & This study \\
\hline pGST-Rv3180c & Rv3180c ${ }^{+}$(toxin), pGST & This study \\
\hline pGST-Rv0299 & Rv0299+ (toxin), pGST & This study \\
\hline pGST-Rv3749c & $R v 3749 c^{+}$(toxin), pGST & This study \\
\hline pMV306AC-Rv2019 & Rv2019+ (toxin), pMV306AC & This study \\
\hline pMV306AC-Rv2018-2019 & $R v 2018^{+}$(antitoxin), Rv2019+ (toxin), pMV306AC & This study \\
\hline pMV306AC-Rv3697c & Rv3697c+ (toxin), pMV306AC & This study \\
\hline pMV306AC-Rv3180c & $R_{v} 3180 c^{+}$(toxin), pMV306AC & This study \\
\hline pMV306AC-Rv3180c-3181c & 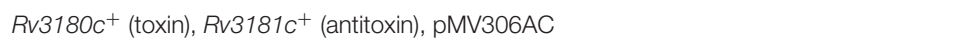 & This study \\
\hline pMV306AC-Rv0299 & Rv0299+ (toxin), pMV306AC & This study \\
\hline pMV306AC-Rv3749c & $R v 3749 c^{+}$(toxin), pMV306AC & This study \\
\hline
\end{tabular}

$A m p^{\mathrm{R}}$, ampicillin resistance; $\mathrm{Kan}^{\mathrm{R}}$, Kanamycin resistance; $\mathrm{Cm}^{\mathrm{R}}$, Chloramphenicol resistance.

centrifugation and resuspended in M9 minimal media to an optical density at $600 \mathrm{~nm}\left(\mathrm{OD}_{600}\right)=0.2$. The resuspension was diluted $10^{-1}, 10^{-2}, 10^{-3}$, and $10^{-4}$-folds, and each diluted sample was spotted onto M9 agar plates containing $\mathrm{Cm}$ and Amp with $0.2 \%$ L-arabinose, $0.05 \mathrm{mM}$ IPTG, or both or without inducers. The plates were incubated overnight at $37^{\circ} \mathrm{C}$. For a neutralization assay in liquid culture, BL21(DE3) harboring pBAD33-Rv2019 and pET21c-Rv2018 were grown at $37^{\circ} \mathrm{C}$ to the exponential phase in M9 minimal media containing $\mathrm{Cm}$ and Amp. The cultures were diluted fivefold in the same minimal media, and inducers were added as follows: $0.2 \% \mathrm{~L}$-arabinose, $0.05 \mathrm{mM}$ IPTG, or both. The diluted cultures were incubated at $37^{\circ} \mathrm{C}$ for $2 \mathrm{~h}$ and continuously diluted fivefold in fresh $\mathrm{M} 9$ minimal media containing inducers every $2 \mathrm{~h}$, and $\mathrm{OD}_{600}$ was measured.

\section{Purification of GST and GST-Rv2019}

Overnight cultures of BL21(DE3) transformed with pGST or pGST-Rv2019 were diluted 50-fold in $1 \mathrm{~L}$ of fresh LB media containing kanamycin and incubated at $37^{\circ} \mathrm{C}$ until $\mathrm{OD}_{600}$ reached $0.5-0.6$. IPTG was added to $0.5 \mathrm{mM}$, and the cultures were further incubated with shaking $(200 \mathrm{rpm})$ at $37^{\circ} \mathrm{C}$ for $2 \mathrm{~h}$. The cells were centrifuged for $20 \mathrm{~min}$ at $4,000 \times g$, and the cell pellet was washed in $30 \mathrm{ml}$ of $20 \mathrm{mM}$ Tris- $\mathrm{HCl}$ (pH 6.8). The washed cells were centrifuged again and resuspended in $20 \mathrm{ml}$ (for GST) or $66 \mathrm{ml}$ (for GST-Rv2019) of lysis buffer [50 mM Tris- $\mathrm{HCl}$ ( $\mathrm{pH} 8.0), 100 \mathrm{mM} \mathrm{NaCl}, 5 \mathrm{mM} \beta$-mercaptoethanol] with (for GST-Rv2019) or without (for GST) sarkosyl ( $N$-lauroylsarcosine). The resuspended cells were lysed using a sonicator. Lysates were centrifuged for $25 \mathrm{~min}$ at $10,000 \times g$, and the supernatant was centrifuged for $1 \mathrm{~h}$ at $180,000 \times g$ in 
a Beckman 70Ti rotor. The final supernatant was loaded onto a glutathione agarose column that was equilibrated with 10 column volumes of lysis buffer. The column was washed with 30 column volumes of lysis buffer, and bound proteins were eluted with $101-\mathrm{ml}$ aliquots of elution buffer containing $10 \mathrm{mM}$ reduced glutathione. The eluted proteins were dialyzed twice overnight against $2 \mathrm{~L}$ of storage buffer [50 mM Tris- $\mathrm{HCl}(\mathrm{pH}$ 8.0), $100 \mathrm{mM} \mathrm{NaCl}$ ].

\section{Extraction of Total RNA}

The hot phenol method was used to extract total cellular RNA (Sarmientos et al., 1983). BL21(DE3) was grown to $\mathrm{OD}_{600}=1.0$, and $1.5 \mathrm{ml}$ of the culture was centrifuged for $2 \mathrm{~min}$ at $17,000 \times \mathrm{g}$. Pelleted cells were resuspended in $500 \mu \mathrm{l}$ of solution A $(20 \mathrm{mM}$ sodium acetate, $10 \mathrm{mM}$ EDTA, $0.5 \%$ SDS), and an equal volume of hot phenol was added, followed by incubation for $10 \mathrm{~min}$ at $60^{\circ} \mathrm{C}$. After centrifugation for $10 \mathrm{~min}$ at $17,000 \times \mathrm{g}, 400 \mu \mathrm{l}$ of the aqueous phase was mixed with $40 \mu \mathrm{l}$ of $3 \mathrm{M}$ sodium acetate ( $\mathrm{pH} 4.3$ ). In total, $1 \mathrm{ml}$ of ice-cold ethanol was added to the mixture, which was incubated for $30 \mathrm{~min}$ at $-20^{\circ} \mathrm{C}$. RNA was collected by centrifugation for $10 \mathrm{~min}$ at $17,000 \times \mathrm{g}$. The pellet was resuspended in $200 \mu \mathrm{l}$ of solution B (20 mM sodium acetate, $10 \mathrm{mM}$ EDTA) and mixed with $20 \mu \mathrm{l}$ of $3 \mathrm{M}$ sodium acetate ( $\mathrm{pH} 4.3$ ). Next, $1 \mathrm{ml}$ of ice-cold ethanol was added, and the sample was incubated for $30 \mathrm{~min}$ at $-20^{\circ} \mathrm{C}$. RNA was collected by centrifugation for $10 \mathrm{~min}$ at $17,000 \times \mathrm{g}$. The pellet was washed with $200 \mu \mathrm{l}$ of $75 \%$ ethanol and dissolved in $50 \mu \mathrm{l}$ of distilled water. The quality of RNA was assessed using agarose gel electrophoresis, and the RNA concentration was determined using Thermo Scientific NanoDrop 2000.

\section{rRNA Cleavage Assay}

For the in vivo assay, BL21(DE3) harboring pBAD33 or pBAD33Rv2019 was grown to $\mathrm{OD}_{600}=0.6-0.7$ in $\mathrm{M} 9$ minimal media containing $\mathrm{Cm}$. The cultures were diluted fivefold in fresh M9 media supplemented with $0.2 \% \mathrm{~L}$-arabinose, and the diluted cultures were incubated at $37^{\circ} \mathrm{C}$ for $2 \mathrm{~h}$. Cultures were continuously diluted fivefold in fresh M9 minimal media containing inducer every $2 \mathrm{~h}$. After induction for $0,2,4$, or $6 \mathrm{~h}$, equal numbers of cells were harvested according to $\mathrm{OD}_{600}$, and the pellets were resuspended in $100 \mu \mathrm{l}$ of $20 \mathrm{mM}$ Tris- $\mathrm{HCl}$ ( $\mathrm{pH}$ 6.8). An equal volume of phenol ( $\mathrm{pH} 8.0$ ) was added, which was vortexed to lyse the cells, and the aqueous phase was electrophoresed through $1.2 \%$ agarose gel. For the in vitro assay, total RNAs were extracted from BL21(DE3), as described earlier. The reaction buffer containing $50 \mathrm{mM}$ Tris- $\mathrm{HCl}(\mathrm{pH} 8.0)$ and the purified protein (15 pmole of GST or GST-Rv2019) was incubated with $2 \mu \mathrm{g}$ of RNA at $37^{\circ} \mathrm{C}$. At the indicated times, reactions were stopped by adding $3 \mu \mathrm{l}$ of $6 \mathrm{x}$ RNA loading buffer [10 mM Tris-HCl (pH 7.5), 0.03\% bromophenol blue, $0.03 \%$ xylene cyanol FF, 60\% glycerol, and $60 \mathrm{mM}$ EDTA]. The samples were electrophoresed through 1.2\% agarose gel, and RNAs were visualized using ethidium bromide.

\section{Quantitative Real-Time PCR (qRT-PCR)}

BL21(DE3) harboring pBAD33 or pBAD33-Rv2019 was grown to the exponential phase, and the culture was diluted 10 -fold in fresh M9 minimal medium containing Cm. L-arabinose was added to the diluted culture to a final concentration of $0.2 \%$. Next, the cultures were incubated with shaking $(200 \mathrm{rpm})$ at $37^{\circ} \mathrm{C}$ for $4 \mathrm{~h}$; the cultures were then diluted 10 -fold in fresh M9 minimal media containing $\mathrm{Cm}$ and $0.2 \% \mathrm{~L}$-arabinose and incubated at $37^{\circ} \mathrm{C}$. After $8 \mathrm{~h}$, the same amounts of cells were harvested, and total RNAs were extracted using the hot phenol method, as described above. RNase-free DNase (Qiagen) was treated to remove contaminating genomic DNA, according to the manufacturer's instructions. After DNase treatment, the quality of total RNA was assessed using agarose gel electrophoresis, and the RNA concentration was determined using Thermo Scientific NanoDrop 2000. Complementary DNA (cDNA) was synthesized using the Superscript III First Strand Synthesis for RT-PCR Kit (Invitrogen) with $1 \mu \mathrm{g}$ of total RNA. The StepOne Plus Realtime PCR System (Applied Biosystems) was used to amplify $1 \mu \mathrm{l}$ of cDNA added to SYBR Green master mix (Qiagen). The eight pairs of primers used for qRT-PCR are listed in Supplementary Table S1. Thermocycling conditions were as follows: initial denaturation for $15 \mathrm{~min}$ at $95^{\circ} \mathrm{C}$, followed by 40 cycles at $95^{\circ} \mathrm{C}$ for $5 \mathrm{~s}, 55^{\circ} \mathrm{C}$ or $59^{\circ} \mathrm{C}$ (only for reaction using the glyS2 primers) for $30 \mathrm{~s}$, and $72^{\circ} \mathrm{C}$ for $50 \mathrm{~s}$.

\section{Construction of $M$. smegmatis Strains Carrying Genomic Expression Cassettes Encoding Toxins}

The shuttle vector pMV306AC lacking a mycobacterial origin of replication was used for integration, which occurs between the attP site of the vector and the attB site of $M$. smegmatis genomic DNA (Stover et al., 1991). Rv2019, Rv2018-2019, $R v 3180 c, R v 3180 c-3181 c, R v 3697 c, R v 3749 c$, or $R v 0299$ was each cloned into the NdeI-XbaI sites of the acetamideinducible pMV306AC vector to generate pMV306AC-Rv2019, pMV306AC-Rv2018-2019, pMV306AC-Rv3180c, pMV306ACRv3180c-3181c, pMV306AC-Rv3697c, pMV306AC-Rv3749c, or pMV306AC-Rv0299, respectively. Electroporation was used to introduce the plasmids into M. smegmatis mc $^{2} 155$ (Snapper et al., 1990). Single colonies of transformed cells were selected by plating the cultures onto agar plates containing kanamycin. Colony PCR was performed to confirm the integration of the plasmid into the bacterial genome (data not shown).

\section{RESULTS}

\section{Assessment of the Toxic Effects of $5 \mathrm{Mtb}$ TA Systems on the Growth of $E$. coli}

The Mtb genome encodes 79 TA systems as follows: $10 \mathrm{MazF}$ homologs, 50 VapC homologs, 2 RelE homologs, 3 HigB homologs, 2 ParE homologs, 1 YoeB homolog, and 11 putative TA systems (Sala et al., 2014). Notably, because of the numerous TA systems in $M t b$, some TA modules were characterized to identify their TA functions; however, the functional mechanisms of most $M t b$ TA systems are unknown. Therefore, in the present study, in order to elucidate functions of those TA systems, we selected 4 VapBC TA systems (Rv2019-Rv2018, 


\section{A}

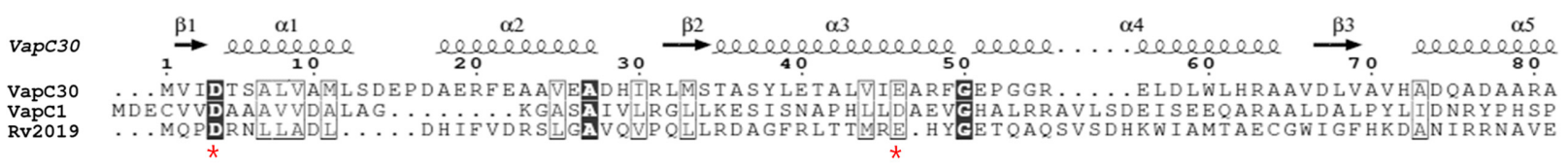

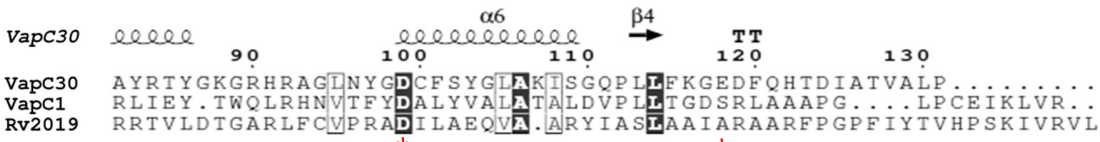

$\mathbf{B}$
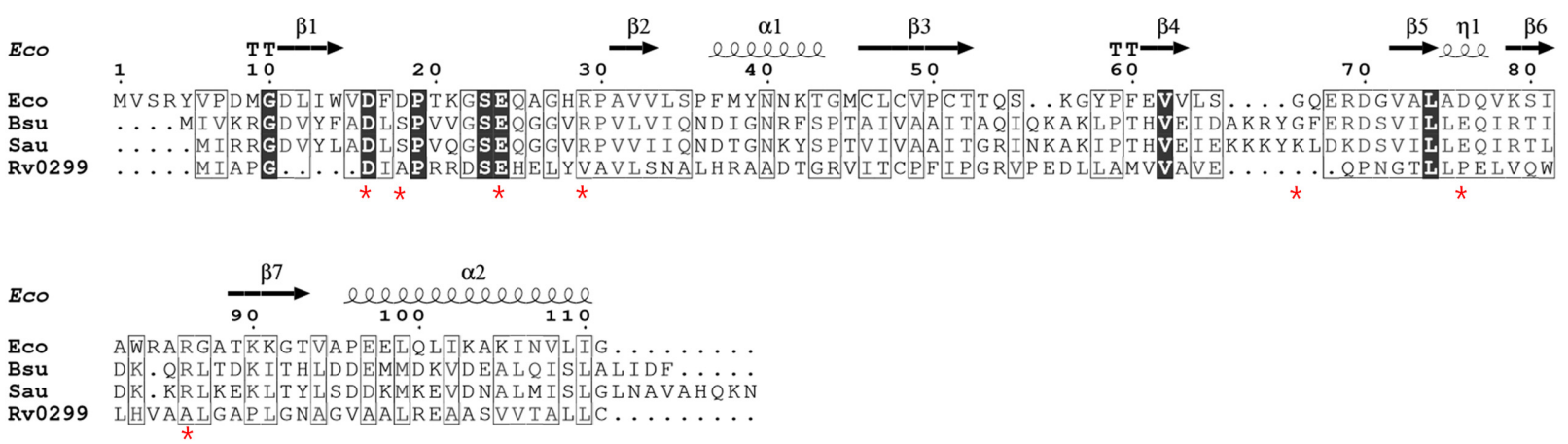

FIGURE 1 | Amino acid sequence alignments of VapC or MazF homologs. (A) Sequence alignments of Rv2019 with VapC homologs VapC30, Mtb VapC30 (GenBank accession number NP_215138.1); VapC1, Mtb VapC1 (CCP42788.1); and Rv2019, Mtb Rv2019 (NP_216535.1). The numbers correspond to amino acid residue numbers and asterisks indicate four highly conserved acidic residues in VapC30. (B) Sequence alignments of Rv0299 with MazF homologs. Eco, Escherichia coli MazF (GenBank accession number CDZ21577.1); Bsu, Bacillus subtilis MazF (P96622.1); Sau, Staphylococcus aureus MazF (AKB00150.1); and Rv0299, Mtb Rv0299 MazF (CCP43029.1). Asterisks indicate seven amino acids residues in E. coli MazF that bind substrate. Identical and similar residues are enclosed in black and white boxes, respectively.

Rv3697c-Rv3697A, Rv3180c-Rv3181c, and Rv3749c-Rv3750c) and 1 MazEF TA system (Rv0299-Rv0298) of Mtb whose toxicities are still unknown in $\mathrm{Mtb}$ as well as in E. coli.

VapC toxin homologs share sequence similarities with the PIN domain, which is a PilT N-terminal domain (Arcus et al., 2011). The three-dimensional structure of the active site of PIN domain includes four highly conserved acidic residues that coordinate the divalent metal ion. Notably, a cluster of metal ion-associated acidic residues forms a cavity and plays an important role in ribonuclease activity (Das et al., 2014). First, we compared the amino acids sequences of four VapC homologs with those of $M t b$ VapC1 and VapC30, each of which contains well-conserved acidic residues. As shown in Figure 1A, the alignment of the amino acids sequence of the Rv2019 toxin with those of the Mtb VapC homologs (VapC1 and VapC30) showed relatively low identities (6.87-10.52\%) and similarities (10.68-20.61\%). Although the overall sequence conservation was poor, the three acidic residues Asp4, Glu41, and Asp98 in Rv2019 were well conserved. However, the fourth acidic residue was not conserved. When the other three VapC homologs (Rv3180c, Rv3697c, and Rv3749c) were aligned with $M t b$ VapC1 and VapC30, three acidic residues were less well conserved (Supplementary Figure S1). All $M t b$ MazF toxins appeared distantly related to known MazF proteins of other bacteria (Ramage et al., 2009), and in the case of Rv0299 toxin as a MazF homolog, the similarity and identity scores of Rv0299 and MazF homologs in E. coli, Bacillus subtilis, and Staphylococcus aureus were 9.9-12.61\% and 5-8\%, respectively (Figure 1B).

To assess the toxic effects of five putative $M t b$ TA systems on E. coli, we transformed BL21(DE3) with the pBAD33-toxin plasmid and induced the expression of toxin genes using $0.2 \% \mathrm{~L}-$ arabinose. The expression of the five mycobacterial TA systems in cells grown on solid M9 media showed only that the expression of Rv2019 inhibited colony formation of BL21(DE3) (Figure 2A). Further, the expression of Rv3180c, Rv3697c, Rv3749c, and Rv0299 did not influence colony formation of BL21(DE3). The open reading frames of $R v 2018, R v 3181 c, R v 3697 A, R v 3750 c$, and $R v 0298$ were ligated to the pET21c vector containing an IPTGinducible (T7) promoter. In the presence of $0.05 \mathrm{mM}$ IPTG, the expression of the antitoxins did not affect cell growth, suggesting that the antitoxin proteins were not toxic. Further, only the Rv2019 toxin severely inhibited the growth of E. coli when each of the five toxins was expressed in cells cultured in liquid M9 media (Figure 2B). These results suggest that $M t b$ Rv2019 functions as a toxin, at least in E. coli.

Next, to investigate whether the three acidic residues are indispensable for the toxicity of the Rv2019 toxin, the mutations $\mathrm{D} 4 \mathrm{~K}, \mathrm{E} 41 \mathrm{~K}$, and $\mathrm{D} 98 \mathrm{~K}$ were individually introduced into the primary sequence of the Rv2019 toxin. In Figure 2C, the expression of the Rv2019 ${ }_{D 4 K}$ mutant inhibited colony formation of BL21(DE3) to an extent similar to that of wild-type Rv2019. In contrast, the Rv2019 $9_{E 41 K}$ and Rv2019 ${ }_{D 98 K}$ mutants did not inhibit 
A

Toxin

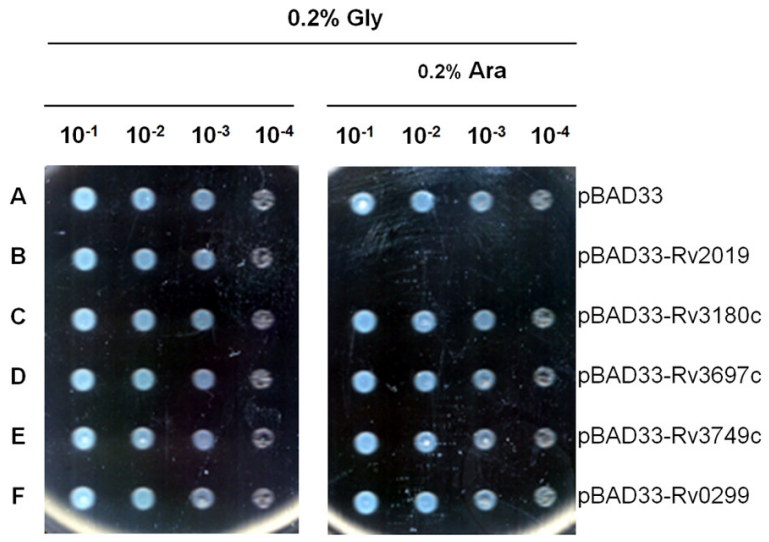

Antitoxin

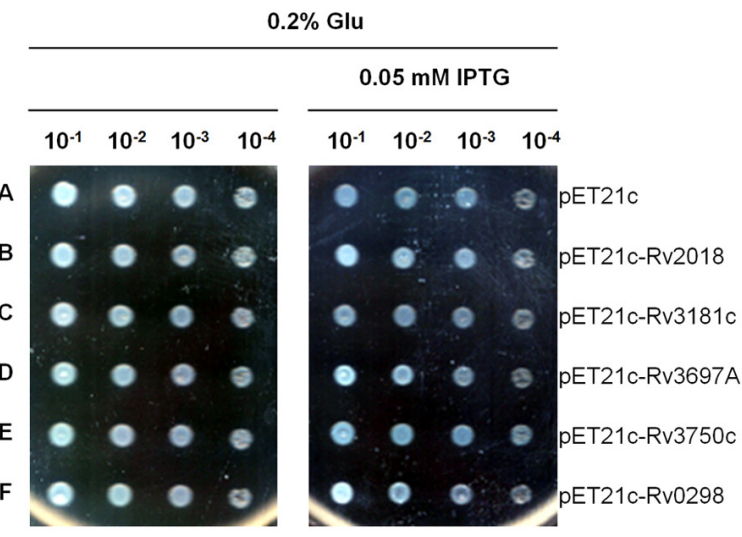

C
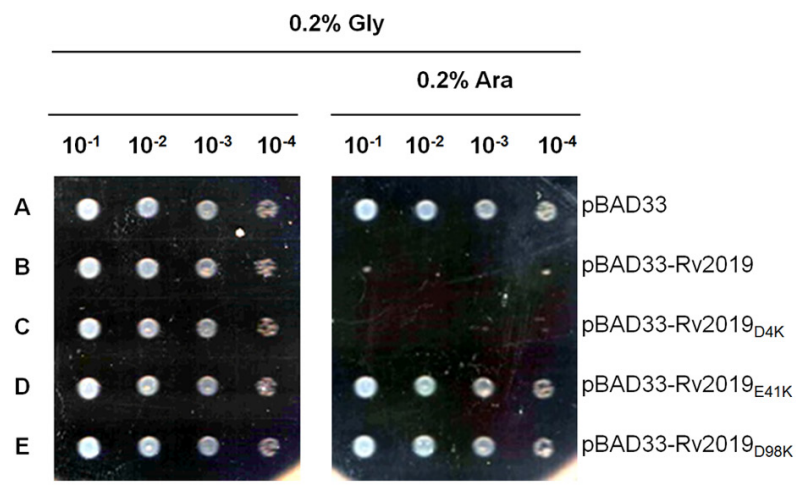

B
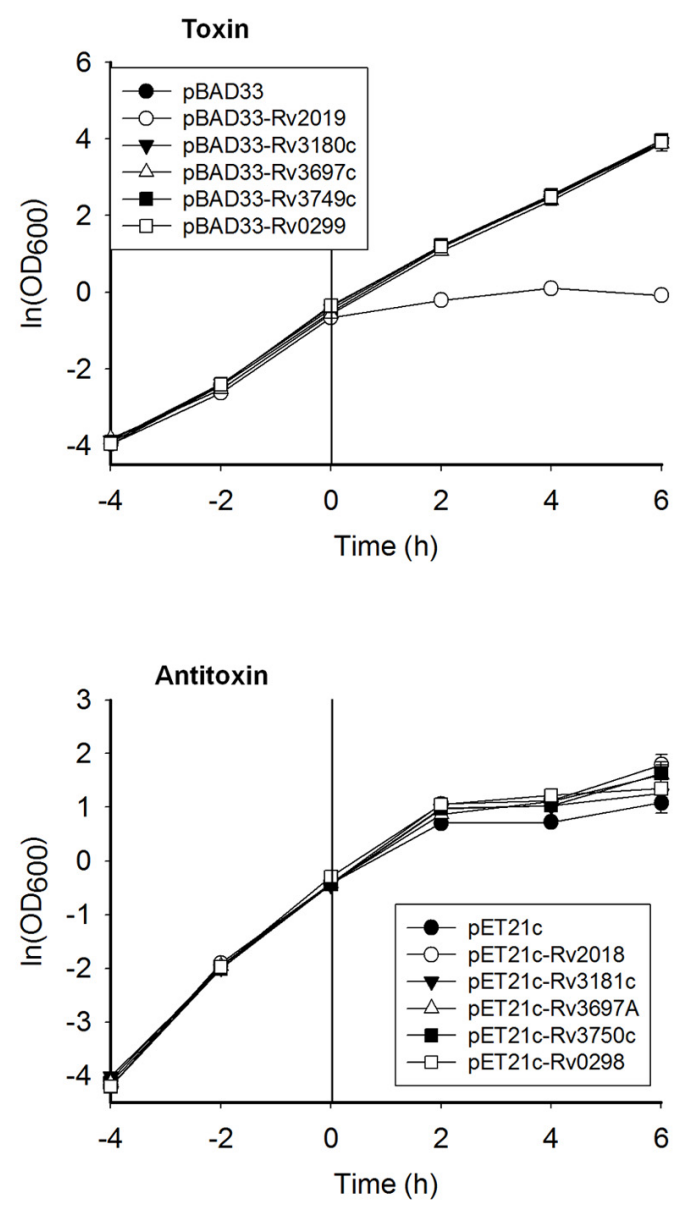

FIGURE 2 | Phenotypes of five putative toxin-antitoxin systems and mutant Rv2019 proteins. (A) Inhibition of colony formation of $E$. coli BL21(DE3) expressing Rv2019 toxin. BL21(DE3) harboring plasmid pBAD33, pBAD33-toxin, pET21c, or pET21c-antitoxin was collected during the exponential phase and harvested by centrifugation. The cell pellet was resuspended to an optical density at $600 \mathrm{~nm}\left(\mathrm{OD}_{600}\right)=0.2 \mathrm{in} \mathrm{M9}$ minimal media and diluted $10^{-1}, 10^{-2}, 10^{-3}$, and $10^{-4}$ folds. Each diluted sample was spotted on M9 agar plates. (B) Growth curves of E. coli BL21(DE3) expressing toxin or antitoxin. BL21(DE3) harboring the plasmids pBAD33, pBAD33-toxin, pET21c, or pET21c-antitoxin was diluted during the exponential phase, and the inducer was added at a time defined as $0 \mathrm{~h}$. Cultures were diluted at every $2 \mathrm{~h}$, and $\mathrm{OD}_{600}$ was measured. (C) Colony formation of $E$. coli BL21(DE3) expressing wild-type and mutant Rv2019 proteins. BL21(DE3) harboring pBAD33, pBAD33-Rv2019, pBAD33-Rv2019D4K, pBAD33-Rv2019 E41K, or pBAD33-Rv2019D98K was spotted on M9 agar plates in a manner similar to that described in (A). 
A

$0.2 \%$ Gly

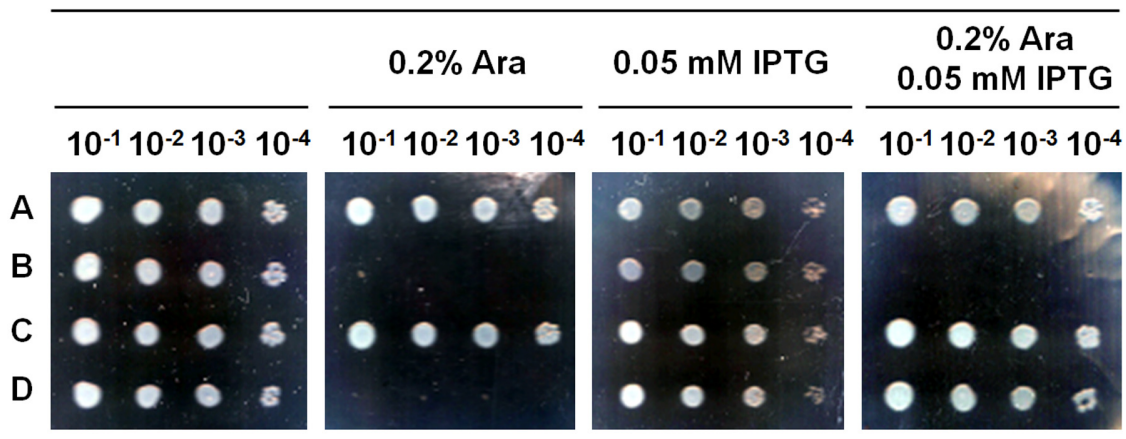
A : pBAD33/pET21c
B : pBAD33-Rv2019/pET21c
C : pBAD33/pET21c-Rv2018
D : pBAD33-Rv2019/pET21c-Rc2018

B

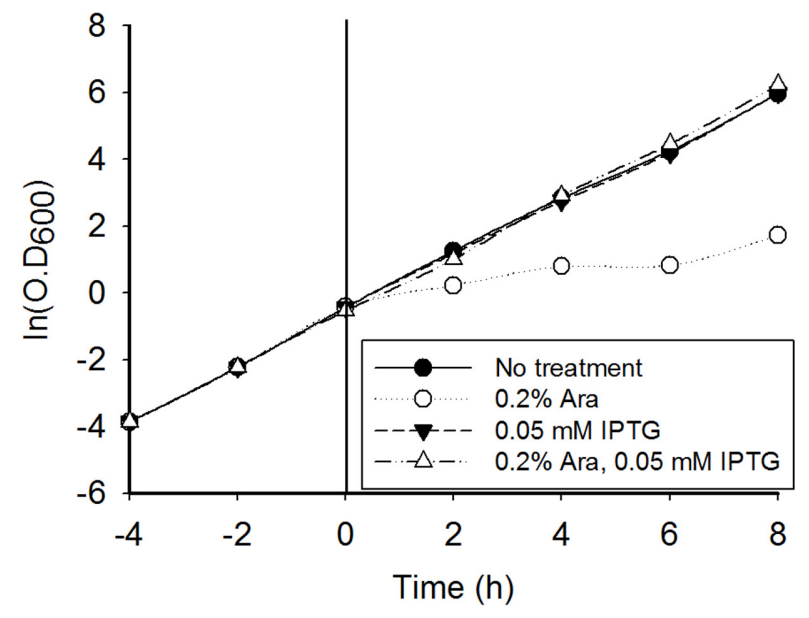

FIGURE 3 | Neutralization of the toxic effect of Rv2019 by the Rv2018 antitoxin. (A) Recovered colony formation of BL21(DE3) coexpressing Rv2019 and Rv2018. BL21(DE3) harboring compatible plasmid pairs (pBAD33 and pET21c, pBAD33-Rv2019 and pET21c, pBAD33 and pET21c-Rv2018, or pBAD33-Rv2019 and pET21c-Rv2018) was diluted during the exponential phase. The cells were harvested by centrifugation, resuspended to $\mathrm{OD}_{600}=0.2$ in $\mathrm{M} 9 \mathrm{minimal}$ media, and diluted $10^{-1}, 10^{-2}, 10^{-3}$, and 10 $10^{-4}$ folds. Each of the diluted samples was spotted on M9 agar plates. (B) Growth curve of BL21(DE3) coexpressing Rv2019 and Rv2018. BL21(DE3) harboring pBAD33-Rv2019 and pET21c-Rv2018 was diluted fivefold in fresh M9 minimal media during the exponential phase, and inducer was added at $0 \mathrm{~h}$. Cultures were continuously diluted fivefold in fresh M9 minimal media containing inducers every $2 \mathrm{~h}$, and $\mathrm{OD}_{600}$ was measured.

colony formation of BL21(DE3). These results suggest that Glu41 and Asp98, but not Asp4, play a pivotal role in the toxicity of Rv2019 and coordination of metal ion and that the toxicity is solely due to the expression of Rv2019.

\section{Neutralization of the Rv2019 Toxin by the Rv2018 Antitoxin}

In exponentially growing healthy cells, all toxins are constitutively expressed and interact with their cognate antitoxins, either protein or RNA molecules, except for the Type IV TA system. This association prevents the function of a toxin by neutralizing its toxicity. Therefore, to examine whether the toxic effect of Rv2019 was neutralized by its cognate Rv2018 antitoxin, E. coli BL21(DE3) was cotransformed with compatible plasmid pairs (pBAD33 and pET21c, pBAD33-Rv2019 and pET21c, pBAD33 and pET21c-Rv2018, or pBAD33-Rv2019 and pET21c-Rv2018). In BL21(DE3) cotransformants, the expression of the gene encoding the Rv2019 toxin or the Rv2018 antitoxin was independently induced by $0.2 \%$ L-arabinose or $0.05 \mathrm{mM}$ IPTG and coinduced when $0.2 \%$ L-arabinose and $0.05 \mathrm{mM}$ IPTG were added together. We found that the expression of the Rv2019 toxin inhibited colony formation of BL21(DE3) in the presence of arabinose (Figure 3A), which is consistent with the previous result (Figure 2A). However, coexpression of the Rv2019 toxin and the cognate Rv2018 
$\mathbf{A}$

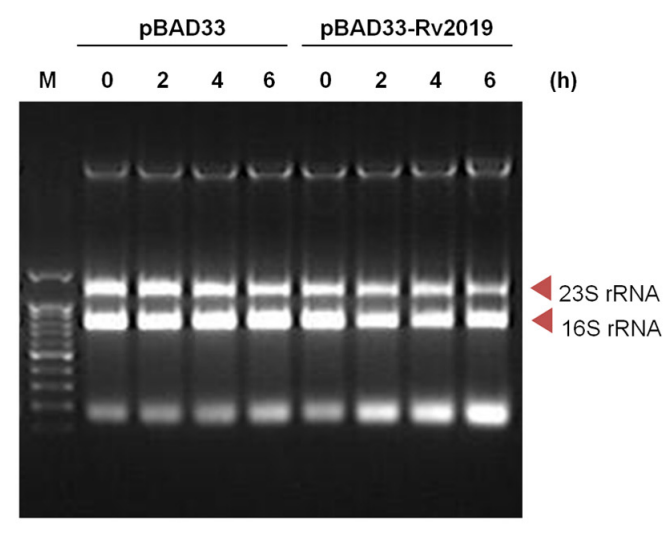

B

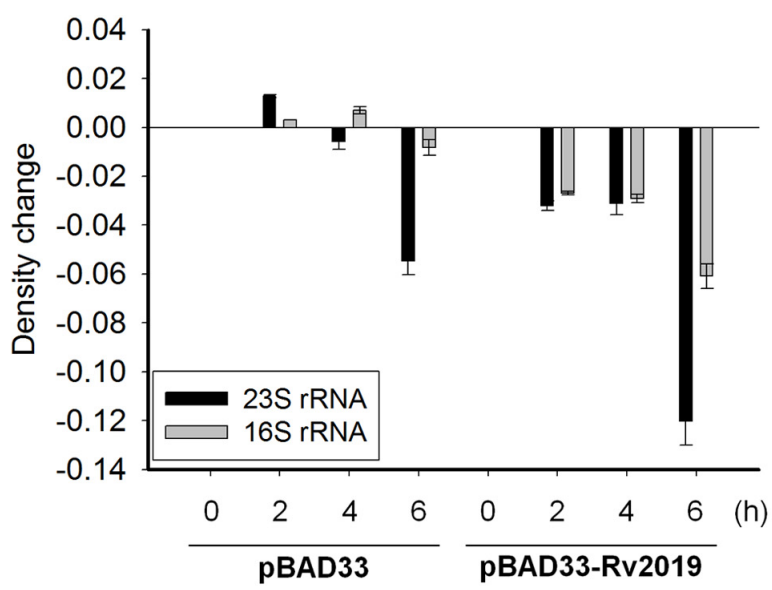

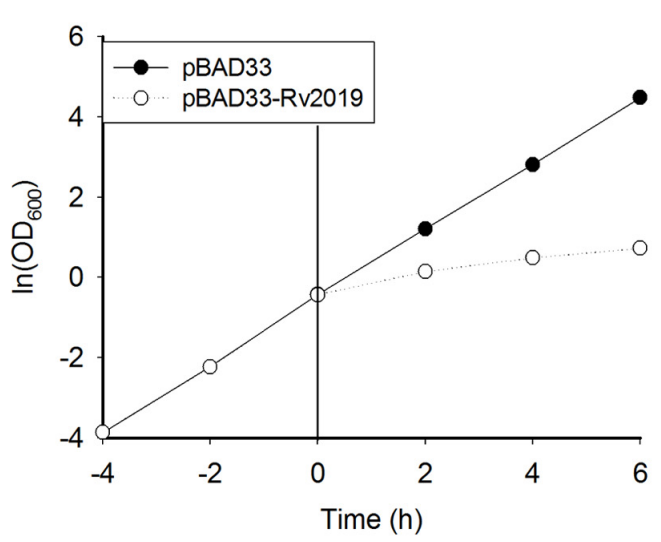

C

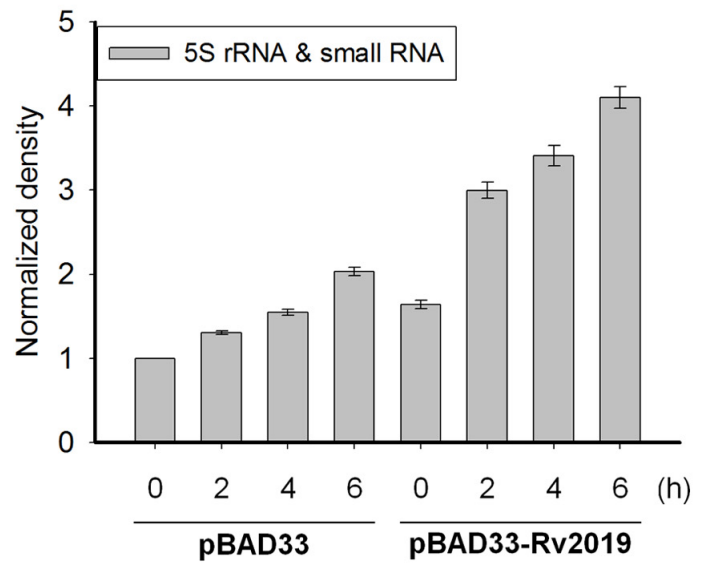

FIGURE 4 | Ribosomal RNA cleavage activity of Rv2019 in vivo. (A) Ribonuclease activity of Rv2019. BL21(DE3) harboring pBAD33 or pBAD33-Rv2019 was cultivated, and toxin induction was performed, as shown in Figure 2B. Total nucleic acids were extracted using phenol, as described in the section "Materials and Methods." Ethidium bromide staining was used to visualize total nucleic acids. (B) Comparison of the relative abundance of $23 \mathrm{~S}$ and $16 \mathrm{~S}$ rRNA bands. The intensities of 23S and 16S rRNA bands were quantified using ImageJ software. The ratio of 23S rRNA/(16S+23S rRNA) and 16S rRNA/(16S+23S rRNA) was calculated and normalized to the band density of $16 \mathrm{~S}+23 \mathrm{~S} \mathrm{rRNA}$ in the first lane $(0 \mathrm{~h})$. The bar graph shows a change of the values from $0 \mathrm{~h}$ to $\mathrm{x} \mathrm{h}(0,2,4$, and $6 \mathrm{~h})$. (C) Relative densities of $5 \mathrm{~S}$ rRNA and small RNA bands. The intensities of $5 \mathrm{~S}$ rRNA and small RNA bands were quantified using Image software, and the values were normalized to the standard in the first lane $(0 \mathrm{~h})$.

antitoxin recovered normal colony formation of BL21(DE3) in the presence of both inducers. Similar results were obtained using liquid cultures (Figure 3B). Furthermore, a reciprocal exchange in expression vectors yielded the same results (Supplementary Figure S2A). Thus, these findings demonstrate that the expression of the Rv2019 toxin is toxic to E. coli and its cognate antitoxin Rv2018 counteracts the toxic effect of the Rv2019 toxin.

\section{Ribonuclease Activity of the Rv2019 Toxin In vivo}

In many bacteria, various VapC homologs exhibit ribonuclease activity in vitro or in vivo (Ramage et al., 2009; Maezato et al., 2011; Winther and Gerdes, 2011; McKenzie et al., 2012a; Winther et al., 2013; Lopes et al., 2014). Therefore, we decided to investigate the ribonuclease activity of the Rv2019 toxin in vivo. First, we determined the amount of and pattern changes of total nucleic acids extracted from cells after inducing the expression of the Rv2019 toxin. The expression of the Rv2019 toxin was induced by adding L-arabinose to exponential phase cells harboring pBAD33 or pBAD33-Rv2019, and $\mathrm{OD}_{600}$ was measured at 2 -h intervals. The same number of cells was harvested at each time point, and total nucleic acids were extracted using phenol. In the presence of L-arabinose, control cells exhibited normal exponential growth, and the level of total nucleic acids remained constant after induction (Figure 4A). However, after the expression of the Rv2019 toxin, we found that $23 \mathrm{~S}$ rRNA level gradually decreased, and that more interestingly, the amount of small RNAs increased for $6 \mathrm{~h}$. The intensities of the $23 \mathrm{~S}$ and $16 \mathrm{~S}$ rRNA and small RNA bands were analyzed using 
A

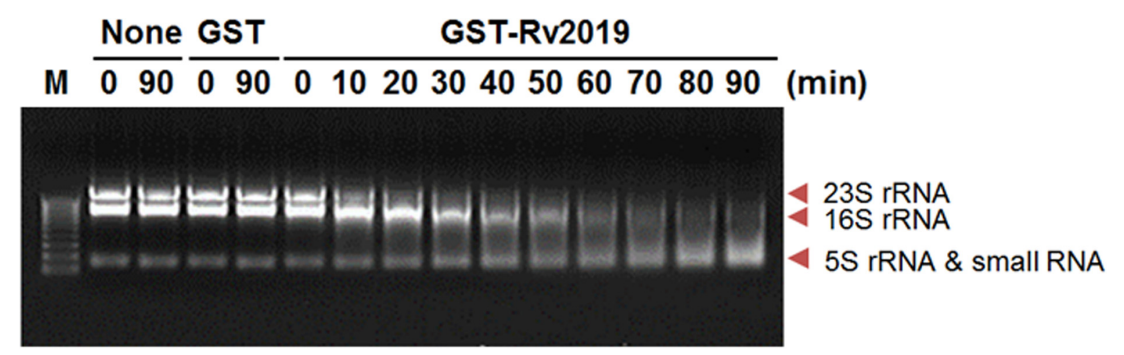

B
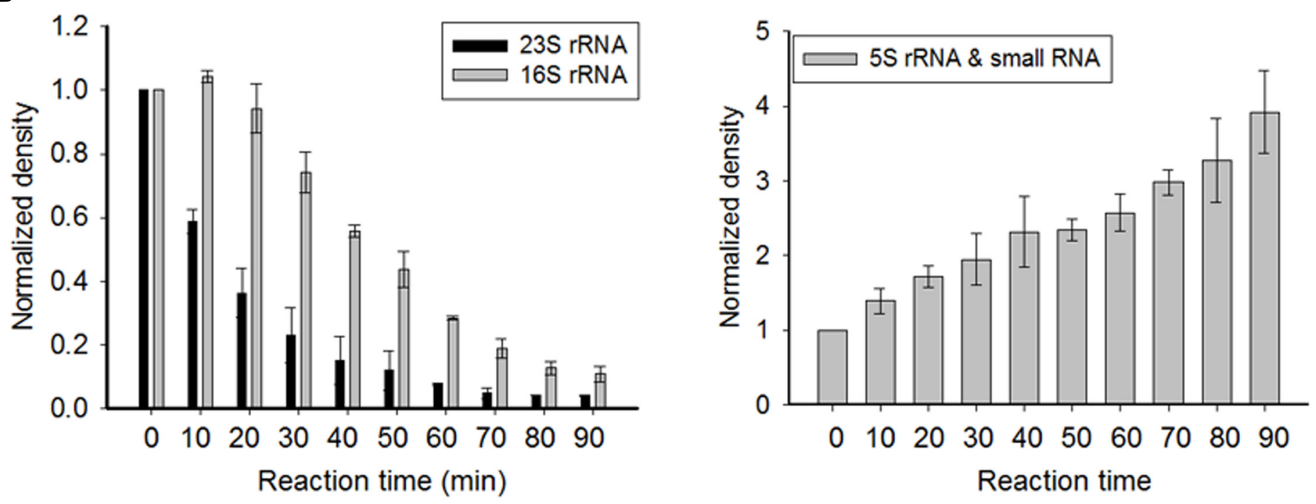

C

No
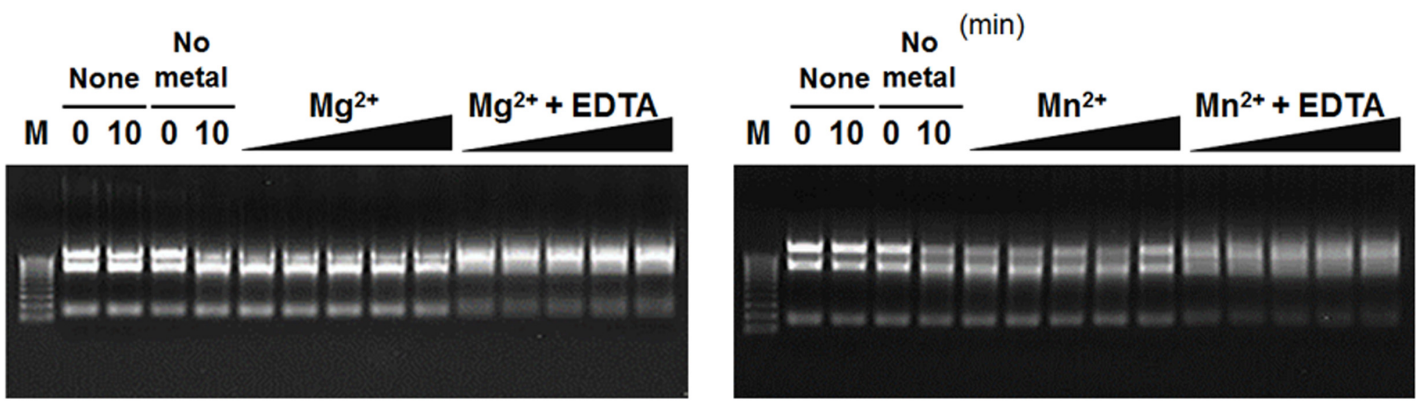

FIGURE 5 | Ribosomal RNA cleavage activity of Rv2019 in vitro. Total RNAs were isolated from E. coli BL21(DE3) using the hot phenol method. In total, 15 pmole of GST-Rv2019 was incubated with $2 \mu \mathrm{g}$ of total RNA in $50 \mathrm{mM}$ Tris-HCl (pH 8.0). (A) Time-dependent ribonuclease activity of Rv2019. GST-Rv2019 was incubated with the substrate for 0 to 90 min. Cleavage assay was independently performed three times. (B) Relative density of RNA bands. Image J software was used for quantification and normalization. (C) Inhibition of ribonuclease activity of Rv2019 by EDTA. GST-Rv2019 was incubated for 15 min with increasing amounts of metal ion $(0.0015,0.015,0.15,1.5$, and $15 \mathrm{mM})$ before the addition of total RNA with or without $60 \mathrm{mM}$ EDTA. The reaction mixture was incubated for 10 min.

Image J software. Figure $4 \mathrm{~B}$ shows that after the induction of the Rv2019 toxin, the levels of $23 \mathrm{~S}$ and $16 \mathrm{~S}$ rRNAs substantially declined after $6 \mathrm{~h}$, and the levels of small RNAs increased (Figure 4C), indicating the accumulation of fragments of $23 \mathrm{~S}$ and $16 \mathrm{~S}$ rRNAs. The amount of genomic DNA at the top of the gel remained constant, indicating the RNA-specific activity of Rv2019. These results suggest that the Rv2019 toxin degrades rRNA and that its activity inhibits the growth of BL21(DE3).

\section{Ribonuclease Activity of Rv2019 Toxin In vitro}

To detect the ribonuclease activity of the Rv2019 toxin in vitro, we cloned pGST-Rv2019, which produces a GST-Rv2019 chimera, and tested its effects on the growth of BL21(DE3) (Supplementary Figure S3B). The coexpression of GST-Rv2019 and Rv2018 recovered the normal growth of BL21(DE3) (Supplementary Figure S3C). Next, we extracted total RNAs from exponentially growing BL21(DE3) and purified GST and GST-fused Rv2019 (GST-Rv2019). Purified GST or GST-Rv2019 (15 pmoles each) was incubated with $2 \mu \mathrm{g}$ of total $E$. coli RNAs for $90 \mathrm{~min}$ at $37^{\circ} \mathrm{C}$ in $50 \mathrm{mM}$ Tris- $\mathrm{HCl}(\mathrm{pH} 8.0)$. As shown in Figure 5A, total E. coli RNAs were cleaved by purified GST-Rv2019, and the activity increased with time. Moreover, RNA was not detectably digested in the absence of GST-Rv2019 or by GST. Notably, the intensity of 23S rRNA abruptly decreased after $10 \mathrm{~min}$; however, $16 \mathrm{~S}$ rRNA began to disappear after $30 \mathrm{~min}$ (Figure 5B, left) with a concomitant increase in the intensities of smaller RNAs (Figure 5B, right). Therefore, the purified Rv2019 toxin degrades 


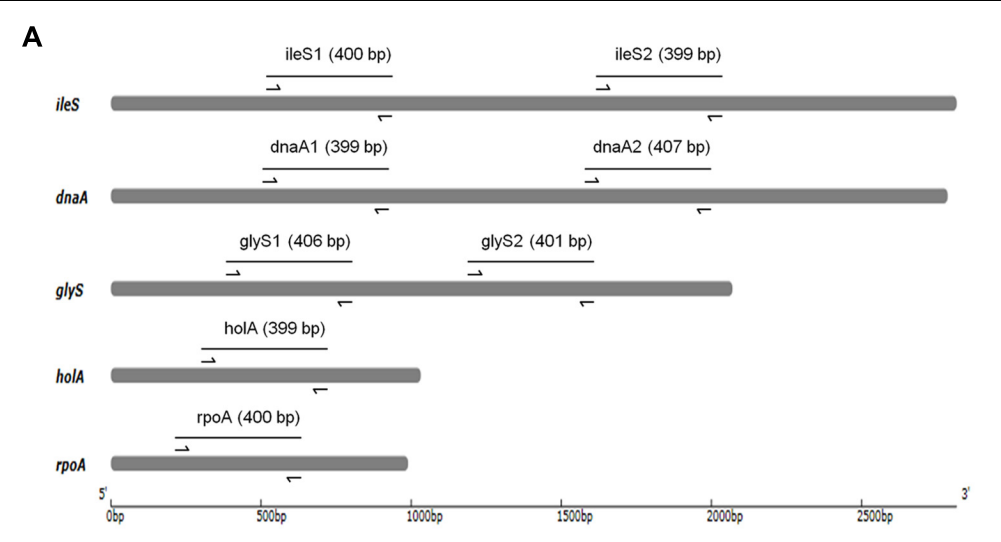

B

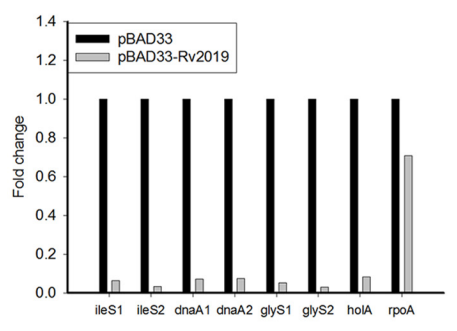

FIGURE 6 | Quantitative real-time PCR analysis of mRNAs with or without induction of Rv2019. (A) Diagram of five genes of $E$. coli BL21(DE3) and amplicons. The arrows indicate the primers used for qRT-PCR analysis, and the lines correspond to amplicons. (B) qRT-PCR analysis of ileS, dnaA, glyS, holA, and rpoA mRNAs. For qRT-PCR, total RNA was extracted from BL21(DE3) harboring pBAD33 (black bars) or pBAD33-Rv2019 (gray bars) after induction for 8 h. qRT-PCR was independently performed three times, as described in the section "Materials and Methods," and a representative result is shown. Data are shown in fold change compared to control (pBAD33).

rRNA in vitro as well as in vivo. Interestingly, the in vitro results showed that the Rv2019 toxin preferentially cleaved 23S rRNA. Unfortunately, although the Rv2018 antitoxin antagonized the activity of the GST-Rv2019 toxin in vivo (Supplementary Figure S3C), the addition of a purified N-terminal His $_{6}$-tagged Rv2018 (antitoxin) to the reaction mixture did not inhibit the ribonuclease activity of the toxin (Supplementary Figure S4A). It is therefore very likely that improper association conditions inhibit the binding of the antitoxin to the toxin, as we confirmed the counteracting activity of His-Rv2018 to GST-Rv2019 in vivo (Supplementary Figure S4B).

The activities of VapC toxins of various bacteria depend on $\mathrm{Mg}^{2+}, \mathrm{Mn}^{2+}$, or both (Ahidjo et al., 2011; McKenzie et al., 2012a,b; Lopes et al., 2014). Therefore, we tested the effects of $\mathrm{Mg}^{2+}$ and $\mathrm{Mn}^{2+}$ on the ribonuclease activity of the Rv2019 toxin. In this assay, the reaction was incubated for $10 \mathrm{~min}$ instead of $90 \mathrm{~min}$ to detect the enhanced activity in the presence of $0.0015,0.015,0.15,1.5$, or $15 \mathrm{mM}$ metal ions. Increasing the concentrations of metal ions did not enhance the catalytic activity, and the $23 \mathrm{~S}$ and $16 \mathrm{~S}$ RNAs were not digested in the presence of EDTA. When we performed the reactions in the presence of $\mathrm{Cu}^{2+}, \mathrm{Ca}^{2+}$, or $\mathrm{Co}^{2+}$, the results were the same (Supplementary Figure S4C). This is likely due to the incorporation of metal ions into the proteins during cultivation and purification, suggesting that metal ions are required for the ribonuclease activity of Rv2019 (Figure 5C).

\section{Effects of Rv2019 on mRNAs}

Next, we determined whether Rv2019 digested mRNAs. For this purpose, we selected the E. coli BL21(DE3) genes (ileS and dnaA, $\sim 3 \mathrm{~kb} ; g l y S, \sim 2 \mathrm{~kb}$; and holA and $r p o A, \sim 1 \mathrm{~kb}$ ) and used qRTPCR to amplify specific regions of each. The expression of the Rv2019 toxin was induced by adding L-arabinose to exponential phase cells harboring pBAD33 or pBAD33-Rv2019, and total RNAs were extracted after induction for $8 \mathrm{~h}$. To amplify specific regions of five mRNAs, we designed primer sets that generate an approximately $\sim 400$-bp amplicon (Figure 6A). The cDNA synthesized from total RNA was subjected to qRT-PCR, and the differences in relative expression for each gene are shown in Figure 6B. The relative mRNA amounts of ileS1, ileS2, dnaA1, dnaA2, glyS1, glyS2, and holA were significantly lower ( $\sim 9$-fold) when the Rv2019 toxin was expressed. However, the transcript amount of rpoA was marginally lower in the presence of the Rv2019 toxin, suggesting that Rv2019 may not cleave the rpoA transcript. Therefore, our results show that ileS, dnaA, glyS, and holA mRNAs serve as substrates for the Rv2019 toxin and that the seven specific regions of these mRNAs contain the sequence cleaved by Rv2019. Based on these results, mRNA was likely digested by the ribonuclease activity of the Rv2019 toxin.

\section{Assessment of the Toxic Effects of $5 \mathrm{Mtb}$ Toxins on M. smegmatis}

So far, we determined the toxicities of five putative $M t b$ toxins to E. coli and found that only the Rv2019 toxin caused toxicity, presumably through its ribonuclease activity. Therefore, we assessed the toxic effects of five candidate $M t b$ TA systems on a non-pathogenic species $M$. smegmatis, which is closely related to $M t b$. For this purpose, we constructed expression vectors in pMV306AC, which harbors an acetamide-inducible promoter and integrates into the attB site of $M$. smegmatis genomic DNA. The resulting plasmids pMV306AC-Rv2019, pMV306AC-Rv2018-2019, pMV306AC-Rv3697c, pMV306ACRv3180c, pMV306AC-Rv3180c-3181c, pMV306AC-Rv0299, and pMV306AC-Rv3749c were individually transformed and integrated into the genome of M. smegmatis, as described in the section "Materials and Methods." The initial transformation results showed that the transformation efficiency of pMV306ACRv2019 was significantly lower than those of the other vectors (Figure 7A, plate 2). However, the transformation of pMV306AC-Rv2018-2019 resulted in the similar colony number with that obtained from control vector (Figure 7A, plate 6). This suggests that transient expression of Rv2019 is toxic 


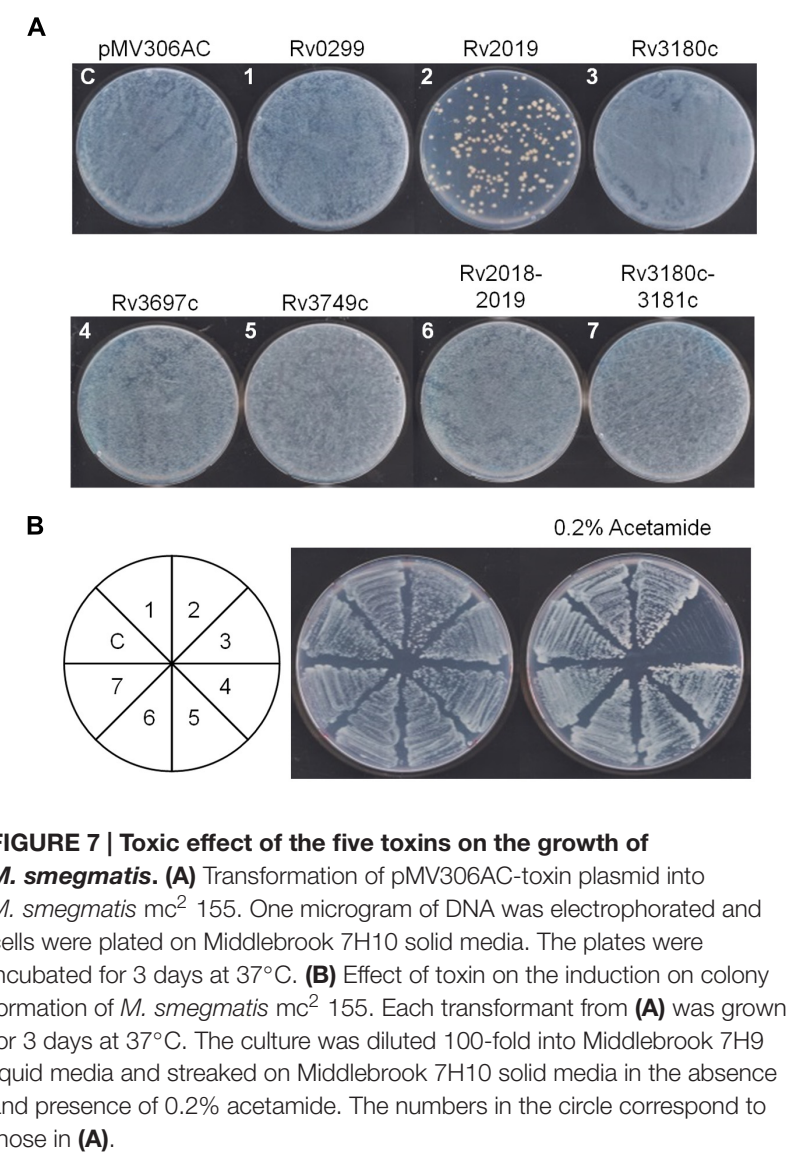

to M. smegmatis and this toxicity was reverted by the expression of antitoxin, Rv2018. Interestingly, when we streaked colonies formed on the initial plates, only the Rv3180c toxin inhibited colony formation of $M$. smegmatis in the presence of $0.2 \%$ acetamide (Figure 7B, sector 3). This toxicity was neutralized by the antitoxin Rv3181c (Figure 7B, sector 7). Further, we found that only the Rv3180c toxin inhibited the growth of M. smegmatis after the addition of acetamide to cells cultured in liquid Middlebrook $7 \mathrm{H} 9$ containing $0.2 \%$ glycerol and $0.02 \%$ Tween 80 (data not shown). Peculiarly, however, the cells transformed with pMV306AC-Rv2019 did not exhibit an inhibitory phenotype upon induction (Figure 7B, sector 2). These findings raise the possibility that mutation, rearrangement, or both of the promoter or the locus harboring $R v 2019$ prevents the expression of Rv2019, and sequencing analysis revealed that transformants of pMV306AC-Rv2019 harbor several spontaneous mutations in the open reading frame of $R v 2019$ (data not shown). Therefore, our results demonstrate that the expression of Rv2019 and Rv3180c inhibited the growth of M. smegmatis, and their cognate antitoxins counteracted the toxicities of toxins.

\section{DISCUSSION}

Mycobacterium tuberculosis possesses 50 putative VapBC TA systems. The structures of the complexes formed by VapBC3,
VapBC5, VapBC15, and VapBC30 have been determined (Miallau et al., 2009; Min et al., 2012; Das et al., 2014; Lee et al., 2015). The active sites of VapC toxins consist of three or four highly conserved acidic residues that bind to $\mathrm{Mg}^{2+}, \mathrm{Mn}^{2+}$, or both, which is a characteristic of the PIN domain. These divalent metal ions coordinate with acidic residues and form a concave active site, thereby helping to stabilize the interaction with negatively charged RNA that is required for RNase activity (Ahidjo et al., 2011; McKenzie et al., 2012a,b; Lopes et al., 2014).

When expressed in E. coli, the VapC homolog Rv2019 toxin, which triggered a growth defect, was neutralized by its cognate antitoxin Rv2018. The Rv2019 toxin appeared to possess ribonuclease activity using free rRNA as a substrate in vivo and in vitro. The Rv2019 toxin cleaved rRNA less effectively in vivo than in vitro, likely due to the association of the rRNA with ribosomal proteins in vivo. Interestingly, the digestion rate of $23 \mathrm{~S}$ rRNA was much faster than that of $16 \mathrm{~S}$ rRNA, indicating more preferred target sites on 23S rRNA. Presumably, this RNase activity is either sequence- or conformation-specific, nevertheless, it is likely that cleavage site specificity of Rv2019 is quite low, resulting in small RNA accumulation. However, this activity was inhibited in the presence of EDTA. Metal ion dependence was further supported by analysis of the mutants Rv2019 ${ }_{E 41 K}$ and Rv2019 ${ }_{D 98 K}$ (Figure 2C). Interestingly, in M. smegmatis, initial transformation of pMV306AC-Rv2019 negatively affected the colony formation, yielding the substantially low transformation efficiency of pMV306AC-Rv2019, meanwhile, this effect was masked by the expression of Rv2018 (Figure 7A). This demonstrates that Rv2019 is paired with Rv2018. Therefore, our findings demonstrate that Rv2019 is a bona fide VapC toxin.

Among five putative toxins, only RV2019 inhibited colony formation after its expression was induced in E. coli, which prompted us to determine the induction level of each recombinant protein. Unfortunately, we were unable to detect significant protein induction using SDS-PAGE (data not shown), nevertheless, the low expression level of Rv2019 was sufficient to cause toxicity.

We next attempted to express GST-fused toxin proteins to determine whether a higher level of expression induced a different phenotype of cell growth. The fusion of each toxin with GST-tag apparently increased the level of induction (Supplementary Figure S3A), and we detected the same toxic phenotype of cells that expressed GST-Rv2019. Notably, serial dilutions of cells that expressed GST-Rv0299 formed less dense colonies, suggesting that GST-Rv0299 was marginally toxic (Supplementary Figure S3B). In E. coli MazF, there are seven amino acid residues, Asp16, Asp18, Glu24, Arg29, Gly66, Asp76, and Arg86 that are involved in the substrate binding, and out of these seven residues, Glu24 and Arg29 are pivotal for catalytic activity of MazF. However, Rv2099 conserved only Asp16 and Glu24 (residue numbers as in E. coli MazF), which might explain the marginal toxicity (Li et al., 2006). Therefore, further studies are required to determine the mechanism of toxicity of Rv2099 as a putative MazF homolog. 
Interestingly, compared with the results shown in Figure 2; Supplementary Figure S3B, the expression of C-terminal His6Rv2019 toxin exhibited a reduced toxic effect (Supplementary Figure S2B). This is probably why the active site is sterically hindered by the His-tag at the C-terminal region which points the incurvate catalytic site (Miallau et al., 2009; Min et al., 2012; Das et al., 2014; Lee et al., 2015).

Type II TA families were originally classified according to their amino acid sequences and three-dimensional structural similarities of their respective toxins (Pandey and Gerdes, 2005). Further, each toxin was paired with its cognate antitoxin depending on their specific interactions, e.g., VapBC and MazEF (Zhang J. et al., 2003; Lopes et al., 2014). Among VapC homologs containing the PIN domain, the majority of toxins interact with antitoxins containing a ribbon-helix-helix (RHH) domain. However, several antitoxins with PHD, AbrB, COG2442, COG2886, MerR, or Xre domains interact with the VapC toxin (Makarova et al., 2009).

In the Rv3180c-Rv3181c TA system, the Rv3180c toxin, which contains a PIN domain, has been predicted to possess ribonuclease activity, and the Rv3181c antitoxin possesses a PHD domain that is recently expected to associate with PIN domain toxins (Makarova et al., 2009). Therefore, the antitoxin containing a PHD domain was considered to interact with a toxin containing the PIN domain. Moreover, it has recently been revealed that a toxin and non-cognate antitoxin interact, and therefore, TA families are independently divided into 12 super-families of Type II toxins and 20 super-families of Type II antitoxins, respectively (Leplae et al., 2011).

There are potentially nine TA systems (one MazF homolog, one VapC homolog, and seven putative TA systems) in M. smegmatis and 33 TA modules in E. coli (Shao et al., 2011; Yamaguchi et al., 2011). It should be noted that neither native nor highly expressed GST-fused Rv3180c was toxic to E. coli (Supplementary Figure S3), suggesting a lack of relationship between expression level and toxicity. Unlike in E. coli, M. smegmatis cells could not form colonies upon induction of Rv3180c.

Therefore, it is possible that Rv3180c may be neutralized by non-cognate antitoxins present in E. coli or that structural conformation of Rv3180c is different in E. coli and M. smegmatis.

\section{REFERENCES}

Ahidjo, B. A., Kuhnert, D., McKenzie, J. L., Machowski, E. E., Gordhan, B. G., Arcus, V., et al. (2011). VapC toxins from Mycobacterium tuberculosis are ribonucleases that differentially inhibit growth and are neutralized by cognate VapB antitoxins. PLoS ONE 6:e21738. doi: 10.1371/journal.pone.0021738

Arcus, V. L., McKenzie, J. L., Robson, J., and Cook, G. M. (2011). The PIN-domain ribonucleases and the prokaryotic VapBC toxin-antitoxin array. Protein Eng. Des. Sel. 24, 33-40. doi: 10.1093/protein/gzq081

Blower, T. R., Short, F. L., Rao, F., Mizuguchi, K., Pei, X. Y., Fineran, P. C., et al. (2012). Identification and classification of bacterial Type III toxin-antitoxin systems encoded in chromosomal and plasmid genomes. Nucleic Acids Res. 40, 6158-6173. doi: 10.1093/nar/gks231

Brzozowska, I., and Zielenkiewicz, U. (2013). Regulation of toxin-antitoxin systems by proteolysis. Plasmid 70, 33-41. doi: 10.1016/j.plasmid.2013.01.007
In the present study, we cloned 5 putative $M t b$ TA modules and expressed them in E. coli and M. smegmatis. Our results revealed that the novel toxin Rv2019 is an RNase that degrades rRNAs and mRNAs and that the Rv2019 and Rv3180c toxins and their cognate Rv2018 and Rv3181c antitoxins functionally associate, respectively. Further studies are required to characterize the other four TA systems. Considering the vast numbers of TA systems of the M. tuberculosis complex, including $M t b$, such studies promise to contribute to a better understanding of the importance of $M t b$ TA systems for controlling latency, persistence, biofilm formation, and stress response.

\section{AUTHOR CONTRIBUTIONS}

YK: executing experiments. EC: writing manuscript. JH: Guiding YK and EC.

\section{FUNDING}

This research was supported by Basic Science Research Program through the National Research Foundation of Korea (NRF) funded by the Ministry of Education, Science and Technology (NRF-2015R1A2A2A01005881). This study was supported by the Research Fund Program of Research Institute for Basic Sciences, Pusan National University, Korea, 2014, Project No. RIBS-PNU2014-01210001.

\section{ACKNOWLEDGMENT}

We thank Dr. Jeong-il Oh for providing Mycobacterium smegmatis mc $^{2} 155$.

\section{SUPPLEMENTARY MATERIAL}

The Supplementary Material for this article can be found online at: http://journal.frontiersin.org/article/10.3389/fmicb. 2016.02071/full\#supplementary-material

Das, U., Pogenberg, V., Subhramanyam, U. K., Wilmanns, M., Gourinath, S., and Srinivasan, A. (2014). Crystal structure of the VapBC-15 complex from Mycobacterium tuberculosis reveals a two-metal ion dependent PIN-domain ribonuclease and a variable mode of toxin-antitoxin assembly. J. Struct. Biol. 188, 249-258. doi: 10.1016/j.jsb.2014.10.002

De la Cruz, M. A., Zhao, W., Farenc, C., Gimenez, G., Raoult, D., Cambillau, C., et al. (2013). A toxin-antitoxin module of Salmonella promotes virulence in mice. PLoS Pathog. 9:e1003827. doi: 10.1371/journal.ppat.1003827

Fineran, P. C., Blower, T. R., Foulds, I. J., Humphreys, D. P., Lilley, K. S., and Salmond, G. P. (2009). The phage abortive infection system, ToxIN, functions as a protein-RNA toxin-antitoxin pair. Proc. Natl. Acad. Sci. U.S.A. 106, 894-899. doi: $10.1073 /$ pnas. 0808832106

Fozo, E. M., Hemm, M. R., and Storz, G. (2008). Small toxic proteins and the antisense RNAs that repress them. Microbiol. Mol. Biol. Rev. 72, 579-589. doi: 10.1128/MMBR.00025-08 
Gerdes, K., Thisted, T., and Martinussen, J. (1990). Mechanism of postsegregational killing by the hok/sok system of plasmid R1: sok antisense RNA regulates formation of a hok mRNA species correlated with killing of plasmid-free cells. Mol. Microbiol. 4, 1807-1818. doi: 10.1111/j.1365-2958.1990. tb02029.x

Guzman, L. M., Belin, D., Carson, M. J., and Beckwith, J. (1995). Tight regulation, modulation, and high-level expression by vectors containing the arabinose PBAD promoter. J. Bacteriol. 177, 4121-4130. doi: 10.1128/jb.177.14.41214130.1995

Hallez, R., Geeraerts, D., Sterckx, Y., Mine, N., Loris, R., and Van Melderen, L. (2010). New toxins homologous to ParE belonging to three-component toxinantitoxin systems in Escherichia coli O157:H7. Mol. Microbiol. 76, 719-732. doi: 10.1111/j.1365-2958.2010.07129.x

Hayes, F. (2003). Toxins-antitoxins: plasmid maintenance, programmed cell death, and cell cycle arrest. Science 301, 1496-1499. doi: 10.1126/science.1088157

Jaffe, A., Ogura, T., and Hiraga, S. (1985). Effects of the ccd function of the F plasmid on bacterial growth. J. Bacteriol. 163, 841-849.

Jiang, Y., Pogliano, J., Helinski, D. R., and Konieczny, I. (2002). ParE toxin encoded by the broad-host-range plasmid RK2 is an inhibitor of Escherichia coli gyrase. Mol. Microbiol. 44, 971-979. doi: 10.1046/j.1365-2958.2002.02921.x

Jorgensen, M. G., Pandey, D. P., Jaskolska, M., and Gerdes, K. (2009). HicA of Escherichia coli defines a novel family of translation-independent mRNA interferases in bacteria and archaea. J. Bacteriol. 191, 1191-1199. doi: 10.1128/ JB.01013-08

Kawano, M., Aravind, L., and Storz, G. (2007). An antisense RNA controls synthesis of an SOS-induced toxin evolved from an antitoxin. Mol. Microbiol. 64, 738-754. doi: 10.1111/j.1365-2958.2007.05688.x

Lee, I. G., Lee, S. J., Chae, S., Lee, K. Y., Kim, J. H., and Lee, B. J. (2015). Structural and functional studies of the Mycobacterium tuberculosis VapBC30 toxinantitoxin system: implications for the design of novel antimicrobial peptides. Nucleic Acids Res. 43, 7624-7637. doi: 10.1093/nar/gkv689

Leplae, R., Geeraerts, D., Hallez, R., Guglielmini, J., Dreze, P., and Van Melderen, L. (2011). Diversity of bacterial type II toxin-antitoxin systems: a comprehensive search and functional analysis of novel families. Nucleic Acids Res. 39, 5513-5525. doi: 10.1093/nar/gkr131

Li, G. Y., Zhang, Y., Chan, M. C., Mal, T. K., Hoeflich, K. P., Inouye, M., et al. (2006). Characterization of dual substrate binding sites in the homodimeric structure of Escherichia coli mRNA interferase MazF. J. Mol. Biol. 357, 139-150. doi: 10.1016/j.jmb.2005.12.035

Liu, M., Zhang, Y., Inouye, M., and Woychik, N. A. (2008). Bacterial addiction module toxin Doc inhibits translation elongation through its association with the 30 S ribosomal subunit. Proc. Natl. Acad. Sci. U.S.A. 105, 5885-5890. doi: 10.1073/pnas.0711949105

Lopes, A. P., Lopes, L. M., Fraga, T. R., Chura-Chambi, R. M., Sanson, A. L., Cheng, E., et al. (2014). VapC from the leptospiral VapBC toxin-antitoxin module displays ribonuclease activity on the initiator tRNA. PLOS ONE 9:e101678. doi: 10.1371/journal.pone.0101678

Maezato, Y., Daugherty, A., Dana, K., Soo, E., Cooper, C., Tachdjian, S., et al. (2011). VapC6, a ribonucleolytic toxin regulates thermophilicity in the crenarchaeote Sulfolobus solfataricus. RNA 17, 1381-1392. doi: 10.1261/rna. 2679911

Makarova, K. S., Wolf, Y. I., and Koonin, E. V. (2009). Comprehensive comparative-genomic analysis of type 2 toxin-antitoxin systems and related mobile stress response systems in prokaryotes. Biol. Direct 4: 19. doi: 10.1186/ 1745-6150-4-19

Masuda, H., Tan, Q., Awano, N., Wu, K. P., and Inouye, M. (2012a). YeeU enhances the bundling of cytoskeletal polymers of MreB and FtsZ, antagonizing the CbtA (YeeV) toxicity in Escherichia coli. Mol. Microbiol. 84, 979-989. doi: 10.1111/j. 1365-2958.2012.08068.x

Masuda, H., Tan, Q., Awano, N., Yamaguchi, Y., and Inouye, M. (2012b). A novel membrane-bound toxin for cell division, CptA (YgfX), inhibits polymerization of cytoskeleton proteins, FtsZ and MreB, in Escherichia coli. FEMS Microbiol. Lett. 328, 174-181. doi: 10.1111/j.1574-6968.2012.02496.x

McKenzie, J. L., Duyvestyn, J. M., Smith, T., Bendak, K., Mackay, J., Cursons, R., et al. (2012a). Determination of ribonuclease sequence-specificity using Pentaprobes and mass spectrometry. RNA 18, 1267-1278. doi: 10.1261/rna. 031229.111
McKenzie, J. L., Robson, J., Berney, M., Smith, T. C., Ruthe, A., Gardner, P. P., et al. (2012b). A VapBC toxin-antitoxin module is a posttranscriptional regulator of metabolic flux in mycobacteria. J. Bacteriol. 194, 2189-2204. doi: 10.1128/JB. 06790-11

Miallau, L., Faller, M., Chiang, J., Arbing, M., Guo, F., Cascio, D., et al. (2009). Structure and proposed activity of a member of the VapBC family of toxinantitoxin systems. VapBC-5 from Mycobacterium tuberculosis. J. Biol. Chem. 284, 276-283. doi: 10.1074/jbc.M805061200

Min, A. B., Miallau, L., Sawaya, M. R., Habel, J., Cascio, D., and Eisenberg, D. (2012). The crystal structure of the Rv0301-Rv0300 VapBC-3 toxin-antitoxin complex from $M$. tuberculosis reveals a $\mathrm{Mg} 2+$ ion in the active site and a putative RNA-binding site. Protein Sci. 21, 1754-1767. doi: 10.1002/ pro. 2161

Munoz-Gomez, A. J., Lemonnier, M., Santos-Sierra, S., Berzal-Herranz, A., and Diaz-Orejas, R. (2005). RNase/anti-RNase activities of the bacterial parD toxinantitoxin system. J. Bacteriol. 187, 3151-3157. doi: 10.1128/JB.187.9.3151-3157. 2005

Ogawa, M., Fujitani, S., Mao, X., Inouye, S., and Komano, T. (1996). FruA, a putative transcription factor essential for the development of Myxococcus xanthus. Mol. Microbiol. 22, 757-767. doi: 10.1046/j.1365-2958.1996.d011725.x

Pandey, D. P., and Gerdes, K. (2005). Toxin-antitoxin loci are highly abundant in free-living but lost from host-associated prokaryotes. Nucleic Acids Res. 33, 966-976. doi: 10.1093/nar/gki201

Ramage, H. R., Connolly, L. E., and Cox, J. S. (2009). Comprehensive functional analysis of Mycobacterium tuberculosis toxin-antitoxin systems: implications for pathogenesis, stress responses, and evolution. PLoS Genet. 5:e1000767. doi: 10.1371/journal.pgen.1000767

Sala, A., Bordes, P., and Genevaux, P. (2014). Multiple toxin-antitoxin systems in Mycobacterium tuberculosis. Toxins (Basel) 6, 1002-1020. doi: 10.3390/ toxins6031002

Sarmientos, P., Sylvester, J. E., Contente, S., and Cashel, M. (1983). Differential stringent control of the tandem E. coli ribosomal RNA promoters from the rrnA operon expressed in vivo in multicopy plasmids. Cell 32, 1337-1346. doi: 10.1016/0092-8674(83)90314-8

Schumacher, M. A., Piro, K. M., Xu, W., Hansen, S., Lewis, K., and Brennan, R. G. (2009). Molecular mechanisms of HipA-mediated multidrug tolerance and its neutralization by HipB. Science 323, 396-401. doi: 10.1126/science. 1163806

Schuster, C. F., and Bertram, R. (2013). Toxin-antitoxin systems are ubiquitous and versatile modulators of prokaryotic cell fate. FEMS Microbiol. Lett. 340, 73-85. doi: 10.1111/1574-6968.12074

Sevin, E. W., and Barloy-Hubler, F. (2007). RASTA-Bacteria: a web-based tool for identifying toxin-antitoxin loci in prokaryotes. Genome Biol. 8:R155. doi: 10.1186/gb-2007-8-8-r155

Shao, Y., Harrison, E. M., Bi, D., Tai, C., He, X., Ou, H. Y., et al. (2011). TADB: a web-based resource for Type 2 toxin-antitoxin loci in bacteria and archaea. Nucleic Acids Res. 39, D606-D611. doi: 10.1093/nar/ gkq908

Snapper, S. B., Lugosi, L., Jekkel, A., Melton, R. E., Kieser, T., Bloom, B. R., et al. (1988). Lysogeny and transformation in mycobacteria: stable expression of foreign genes. Proc. Natl. Acad. Sci. U.S.A. 85, 6987-6991. doi: 10.1073/pnas. 85.18.6987

Snapper, S. B., Melton, R. E., Mustafa, S., Kieser, T., and Jacobs, W. R. Jr. (1990). Isolation and characterization of efficient plasmid transformation mutants of Mycobacterium smegmatis. Mol. Microbiol. 4, 1911-1919. doi: 10.1111/j.13652958.1990.tb02040.x

Stover, C. K., de la Cruz, V. F., Fuerst, T. R., Burlein, J. E., Benson, L. A., Bennett, L. T., et al. (1991). New use of BCG for recombinant vaccines. Nature 351, 456-460. doi: 10.1038/351456a0

Unterholzner, S. J., Poppenberger, B., and Rozhon, W. (2013). Toxin-antitoxin systems: biology, identification, and application. Mob. Genet. Elements 3:e26219. doi: $10.4161 / \mathrm{mge} .26219$

Van Melderen, L., Bernard, P., and Couturier, M. (1994). Lon-dependent proteolysis of CcdA is the key control for activation of CcdB in plasmid-free segregant bacteria. Mol. Microbiol. 11, 1151-1157. doi: 10.1111/j.1365-2958. 1994.tb00391.x 
Vogel, J., Argaman, L., Wagner, E. G., and Altuvia, S. (2004). The small RNA IstR inhibits synthesis of an SOS-induced toxic peptide. Curr. Biol. 14, 2271-2276. doi: 10.1016/j.cub.2004.12.003

Wang, X., Lord, D. M., Cheng, H. Y., Osbourne, D. O., Hong, S. H., SanchezTorres, V., et al. (2012). A new type V toxin-antitoxin system where mRNA for toxin GhoT is cleaved by antitoxin GhoS. Nat. Chem. Biol. 8, 855-861. doi: $10.1038 /$ nchembio. 1062

Weaver, K. E., Ehli, E. A., Nelson, J. S., and Patel, S. (2004). Antisense RNA regulation by stable complex formation in the Enterococcus faecalis plasmid pAD1 par addiction system. J. Bacteriol. 186, 6400-6408. doi: 10.1128/JB.186. 19.6400-6408.2004

Winther, K. S., Brodersen, D. E., Brown, A. K., and Gerdes, K. (2013). VapC20 of Mycobacterium tuberculosis cleaves the sarcin-ricin loop of $23 \mathrm{~S}$ rRNA. Nat. Commun. 4: 2796. doi: 10.1038/ncomms3796

Winther, K. S., and Gerdes, K. (2011). Enteric virulence associated protein VapC inhibits translation by cleavage of initiator tRNA. Proc. Natl. Acad. Sci. U. S. A. 108, 7403-7407. doi: 10.1073/pnas.1019587108

Yamaguchi, Y., Park, J. H., and Inouye, M. (2011). Toxin-antitoxin systems in bacteria and archaea. Annu. Rev. Genet. 45, 61-79. doi: 10.1146/annurev-genet110410-132412
Zhang, J., Zhang, Y., and Inouye, M. (2003). Characterization of the interactions within the mazEF addiction module of Escherichia coli. J. Biol. Chem. 278, 32300-32306. doi: 10.1074/jbc.M304767200

Zhang, Y., Zhang, J., Hoeflich, K. P., Ikura, M., Qing, G., and Inouye, M. (2003). MazF cleaves cellular mRNAs specifically at ACA to block protein synthesis in Escherichia coli. Mol. Cell 12, 913-923. doi: 10.1016/S1097-2765(03)00402-7

Zhang, Y., and Inouye, M. (2011). RatA (YfjG), an Escherichia coli toxin, inhibits $70 \mathrm{~S}$ ribosome association to block translation initiation. Mol. Microbiol. 79, 1418-1429. doi: 10.1111/j.1365-2958.2010.07506.x

Conflict of Interest Statement: The authors declare that the research was conducted in the absence of any commercial or financial relationships that could be construed as a potential conflict of interest.

Copyright (c) $2016 \mathrm{Kim}$, Choi and Hwang. This is an open-access article distributed under the terms of the Creative Commons Attribution License (CC BY). The use, distribution or reproduction in other forums is permitted, provided the original author(s) or licensor are credited and that the original publication in this journal is cited, in accordance with accepted academic practice. No use, distribution or reproduction is permitted which does not comply with these terms. 